\title{
Analytic model for pulsed thermography of subsurface defects
}

by $P$. Broberg *

* University West, SE-46186, Trollhättan, Sweden, patrik.broberg@hv.se

\begin{abstract}
An analytic solution to the heat equation is used to model the response of subsurface defects in pulsed thermography. The model is compared to measurement data and shows good agreement, both in spatial and temporal domain. The capability of the model is then demonstrated by calculating the response of arbitrary defects at different depth. This model, even though simplified, can prove useful due to good accuracy and low computational time for comparing analysis methods and for evaluating a thermography method on a new material or new type of defect.
\end{abstract}

\section{Introduction}

Pulsed thermography is a non-destructive testing method which is today used mainly for composites [1], ceramics [2] and plastics [3], although applications for metals also exists [2-4]. In pulsed thermography a short pulse of energy, usually from a flash lamp, is used for heating the surface of the test piece [5]. This heat is conducted into the material in accordance with the heat equation. If any defect exists under the surface it will block the heat flow and an area of higher temperature, compared to a defect free area, is created above the defect. If the surface temperature is monitored with an IR camera after the pulse, it is possible to detect defects as areas with increased temperature. Since the heat flow is diffusive and spreads out in all directions, the response from a sub-surface defect will be unclear. This makes determining the size and shape of a defect more difficult. The amplitude of the response also decreases with depth, which makes defects with a diameter smaller than the depth difficult to detect.

One way of gaining more knowledge of a method, and to evaluate if it is suitable for a certain application, is to use modelling. As thermography gains acceptance as a non-destructive testing method, it becomes increasingly important to have accurate methods for modelling. It is possible to model thermography using finite element methods [6]; however this is generally computationally heavy, especially if the response is calculated at several times and the excitation is a transient as in pulsed thermography. Another way of modelling heat conduction is to use an analytic solution. This is usually faster although there are often limits in the complexity and geometry of the material. There are several advantages of using modelling instead of experiments, in terms of speed and cost, when it comes to evaluating a thermography method on a new material or new type of defect, as well as for comparing different evaluation methods [7].

\section{Theory}

Starting with the heat equation

$$
\frac{\partial U}{\partial t}=\alpha \nabla^{2} U
$$

where $U$ is the energy density for a homogeneous material and $\alpha$ is the thermal diffusivity, defined as $\alpha=\rho / C_{p} k$. $\rho$ is here the density, $C_{p}$ the specific heat capacity and $k$ the thermal conductivity. This equation can be solved, for a semiinfinite space, in the Fourier domain using the initial condition

$$
U(x, y, z, t=0)=\phi(x, y, z)
$$

where $\phi(r)$ is the energy distribution at time $t=0$ and $r$ is the spatial coordinates. The solution to Eq. (1) for 3dimensional heat conduction is given by

$$
U(x, y, z, t)=\phi(x, y, z) *\left(\frac{1}{4(\pi \alpha t)^{3 / 2}} e^{-\frac{x^{2}+y^{2}+z^{2}}{4 \alpha t}}\right)
$$

This solution is only valid if the material is homogeneous and not in the presents of any defects. In order to have homogenous material properties, only the heat conduction down to the defect is considered. The defect can instead be modelled as a reflector of heat [8]. The heat density at the surface of the test piece can then be separated into two parts, $U_{B}$ and $U_{D}$. The first part, $U_{B}$, is the response of a defect free, semi-infinite, material from the initial pulse. This is here regarded as the background since it does not contain any information about the defect. If the whole surface is heated evenly by the initial pulse, the heat conduction will be 1-dimentional and the background is given by 


$$
U_{B}(t)=\frac{\phi_{0}}{\sqrt{4 \pi \alpha t}}
$$

where $\phi_{0}$ is the amplitude of the pulse. Since this part of the solution does not contain any information about the defect, it is often subtracted during a thermography inspection. The second part, $U_{D}$, is the heat reflected in the defect. If the size and shape of the defect is given by $\phi_{D}$, which also has amplitude $\phi_{0}$, then the contribution from the defect, at the surface of the test piece, is given by

$$
U_{D}(x, y, t)=\frac{e^{-\frac{d^{2}}{\alpha t}}}{4(\pi \alpha t)^{3 / 2}} \cdot\left(\phi_{D}(x, y) * e^{-\frac{x^{2}+y^{2}}{4 \alpha t}}\right)
$$

where $d$ is the depth of the defect. Naturally the heat from the defect will also be reflected in the surface of the test piece and in turn in the defect again, but the contribution from these additional reflections is generally small if the defect is not large and close to the surface. The model here will therefore use only one reflection, but additional ones can be added if needed. There are also other contributions to the response from a defect, such as diffusion around the edge of the defect [9], but these are omitted in this model. Eq. (5) consists of two terms. The first term,

$$
\frac{e^{-\frac{d^{2}}{\alpha t}}}{4(\pi \alpha t)^{3 / 2}}
$$

affects the amplitude of the response from a defect and is dependent on the depth of the defect, and the second

$$
\phi_{D}(x, y) * e^{-\frac{x^{2}+y^{2}}{4 \alpha t}}
$$

makes the signal from the defect less sharp as time increases. Eq. (7) is a convolution between $\phi_{D}$, describing the shape of the defect, and a point spread function, with the same shape as a Gaussian filter, which increases in width as time increases.

An analytical solution, at $z=0$, for a semi-infinite plate containing a defect and with an initial pulse as heating can then be written as the sum of Eq. (4) and Eq. (5).

$$
U(x, y, t)=\frac{e^{-\frac{d^{2}}{\alpha t}}}{4(\pi \alpha t)^{3 / 2}} \cdot\left(\phi_{D}(x, y) * e^{-\frac{x^{2}+y^{2}}{4 \alpha t}}\right)+\frac{\phi_{0}}{\sqrt{4 \pi \alpha t}}
$$

Using Eq. (8) it is possible to estimate the response from an arbitrarily shaped defect, at any time after the heat pulse, for this simplified geometry.

\section{Experimental setup}

Experiments were carried out in order to validate the model, using a $4 \mathrm{~mm}$ steel plate with a flat bottom hole that had a diameter of $9.5 \mathrm{~mm}$ and a depth of $0.75 \mathrm{~mm}$. The material properties were retrieved from a table and adjusted until there was a good compliance.

The thermography system used for the validation experiment consisted of a FLIR SC 5650 IR camera with a band width of $2.5-5.1 \mu \mathrm{m}$ and a $27 \mathrm{~mm}$ optical lens. The camera had an InSb detector with a resolution of $640 \times 512$ pixels and a frame rate of $100 \mathrm{~Hz}$. The flashlamp used delivered a $10 \mathrm{~ms}$, broadband pulse with a total energy of $6 \mathrm{~kJ}$. The distance between the flash lamp and the test piece was $0.16 \mathrm{~m}$, the same as the distance between the camera and the test piece. Both the camera and flash lamp were at a slight angle to the normal of the test piece. The experimental set-up can be seen schematically in figure 1. 


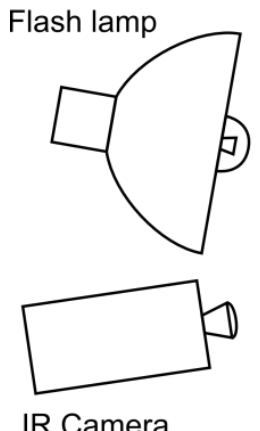

IR Camera

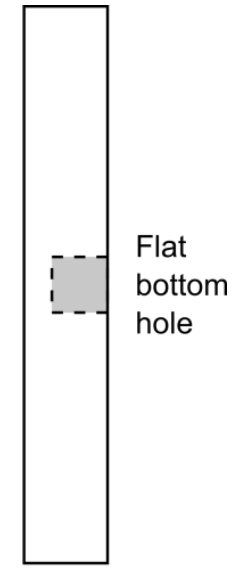

Test piece

Fig. 1. Schematic drawing of the experimental setup.

\section{Results and discussion}

The model in Eq. (8) was implemented using Matlab®. First an image representing the defect $\phi_{D}$ was created. Each pixel in this image was multiplied with Eq. (6) to form an image sequence with the effect of the defect depth. The Gaussian point spread function from Eq. (7) was then calculated for each time step in the sequence. Each image in the sequence was then convoluted with this point spread function using a 2-dimensional convolution. If the background is of interest it is added in the end to the whole image sequence.

The comparison between experimental data and the model is presented in figure 2. It can be seen that all three parts of Eq. (8) show good agreement to the measured data. Eqs. (4), (6) and (7) correspond to the left, middle and right plots in figure 2 respectively. The correlation between the model and experimental data was similar for all points in time during the experiment, although the signal to noise ratio decreased as time increased.
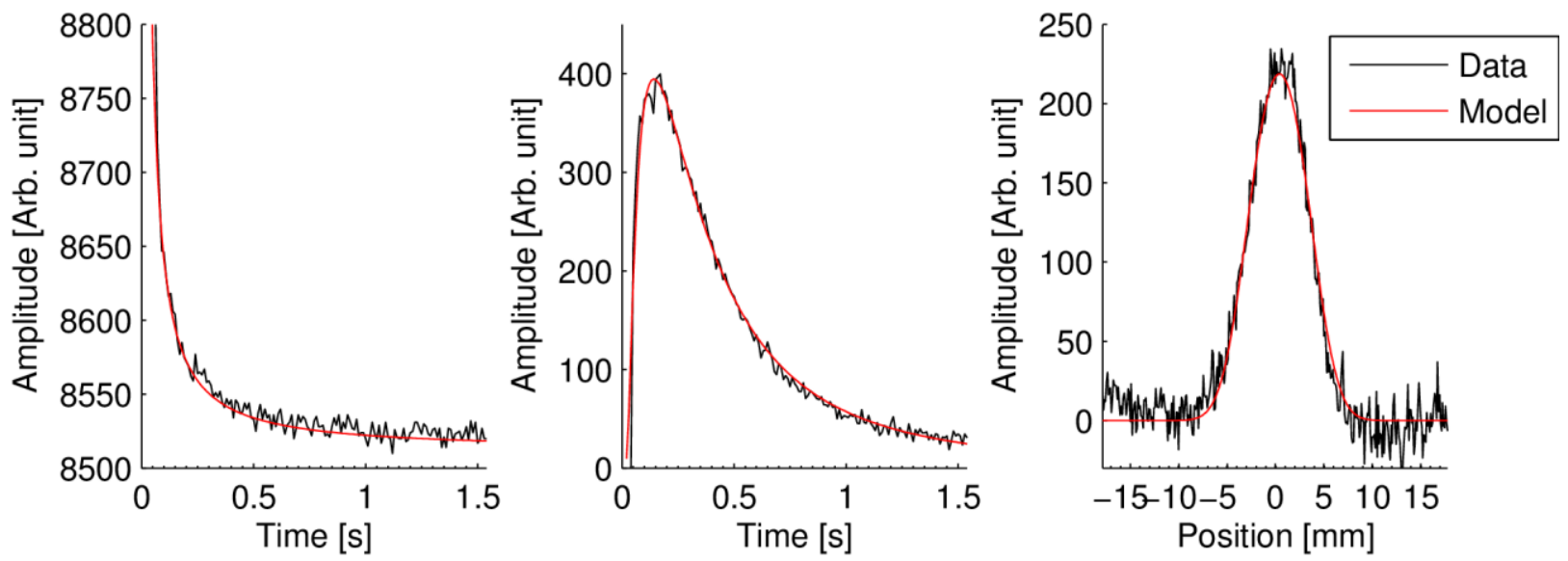

Fig. 2. A comparison between the model and measured data shows good agreement. The left plot shows the background signal in the absence of a defect. The middle plot shows the response of a defect with the background removed. The right plot shows a cross section of the defect $400 \mathrm{~ms}$ after the flash, also with the background removed.

Using this model it is possible to model the response of defects of different sizes, shapes and depths in different materials. Figure 3 shows the response from a square defect, with a width of $10 \mathrm{~mm}$ at a depth of $1.5 \mathrm{~mm}$, in a mild steel plate. In order to have a clearer view of the defect, the background has been removed. The initial heat pulse is at time $t=0$ and as can be seen, the maximum response is about $300 \mathrm{~ms}$ after the initial heating. It can also be seen that the shape of the defect quickly disappears as time increases. The reason for this can be found in the point spread function in Eq. (7), which is a Gaussian function which increases in width as time increases. 


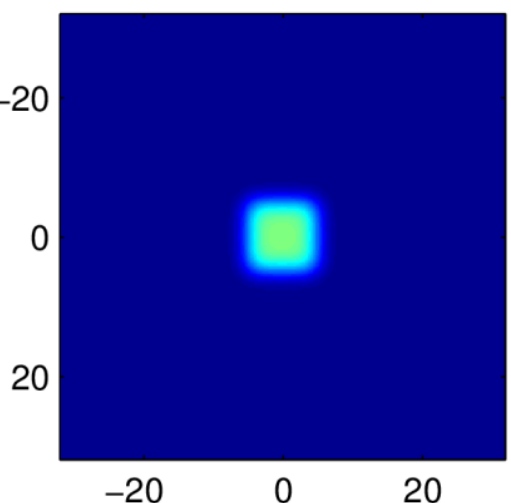

a) Time $100 \mathrm{~ms}$

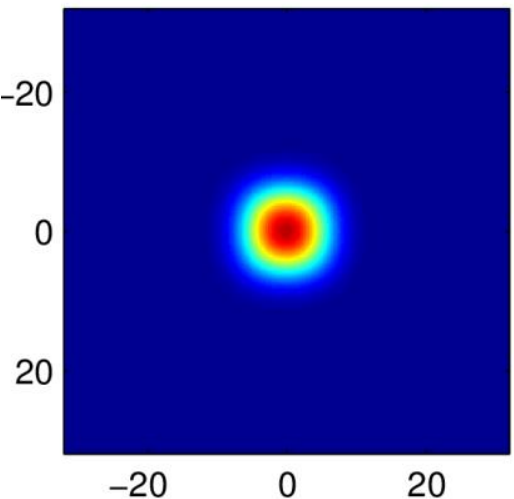

d) Time $400 \mathrm{~ms}$

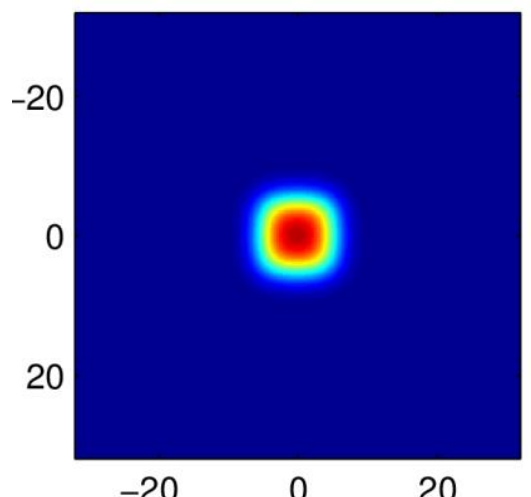

b) Time $200 \mathrm{~ms}$

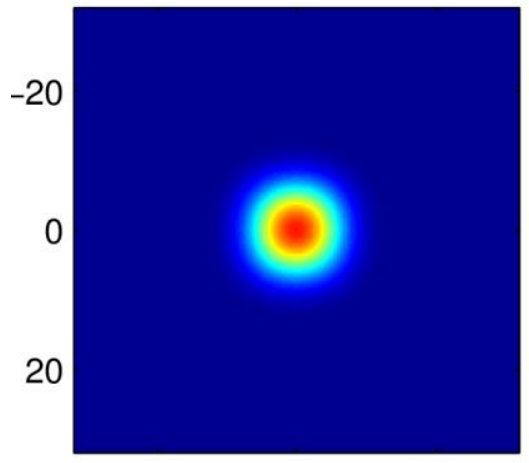

$-20$
0

20

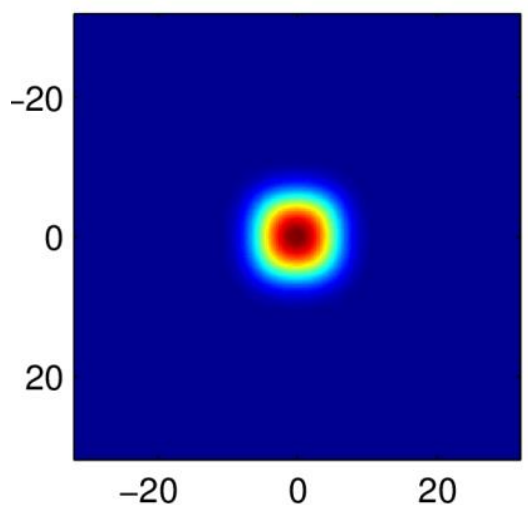

c) Time $300 \mathrm{~ms}$

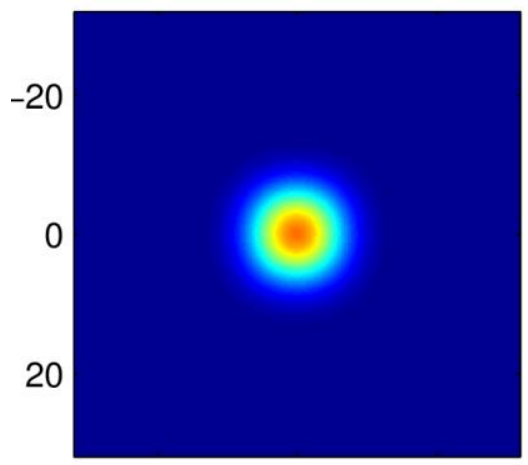

$-20 \quad 20$

f) Time $600 \mathrm{~ms}$

Fig. 3. The responses from a square, $10 \mathrm{~mm}$ in width, defect at different points in time. The scale on the $x$ and $y$-axis is in $\mathrm{mm}$.

To demonstrate the possibilities of modelling arbitrarily shaped defects the response from three defects with the shape of the letters $A, B$ and $C$, as seen in figure $4 a$, were calculated and the result is presented in figure $4 b-d$. The depths of the defects were $0.5,1.0$ and $1.5 \mathrm{~mm}$ respectively for the $A, B$ and $C$. The material properties used in the calculations were those of mild steel. Here it is even clearer that the shape of the defects becomes less distinct as time increases. It can be seen that two defects that are close to each other will become difficult to separate after a short time. Comparing the letters $\mathrm{A}$ and $\mathrm{C}$, which are at different depths, it is clear that the depth affects the amplitude of the response greatly which is why it is difficult to detect defects smaller than the depth.

During a real thermography inspection noise is added to each image, as can be seen in the experimental data in figure 2. To model this, it is possible to add a noise term to Eq. (8), either random noise or a more accurate noise model. 


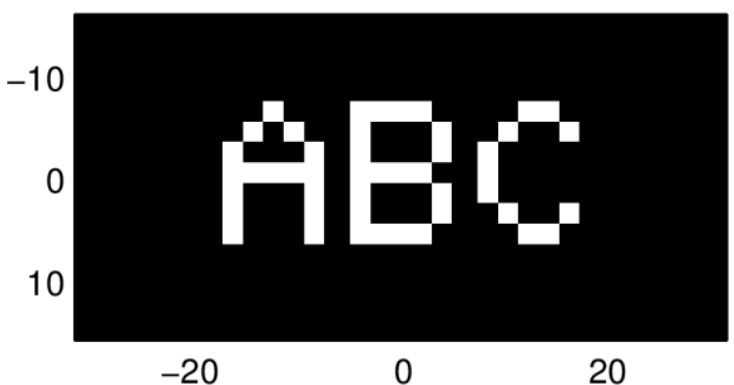

a) Shapes of the defects

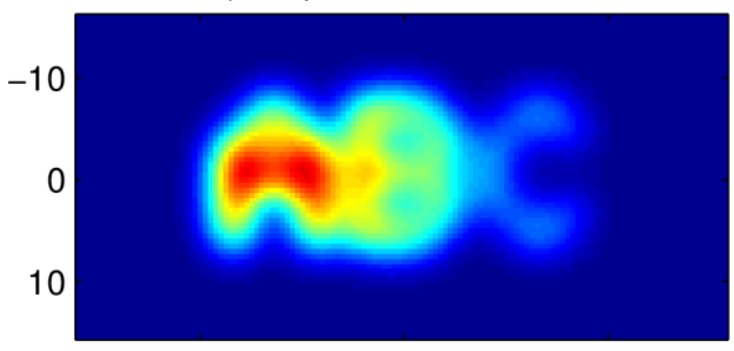

$-20$

0

20

c) Time $200 \mathrm{~ms}$

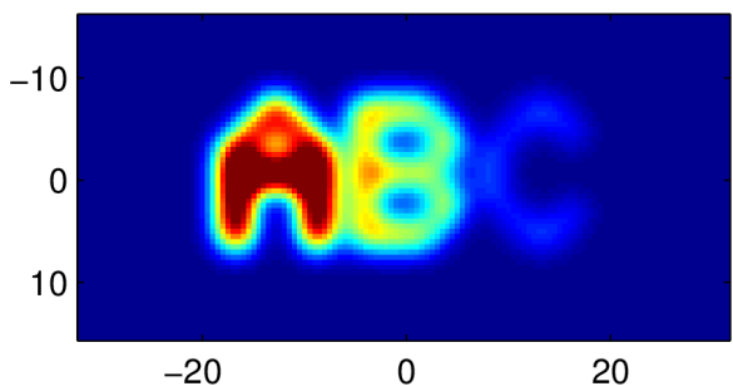

b) Time $100 \mathrm{~ms}$

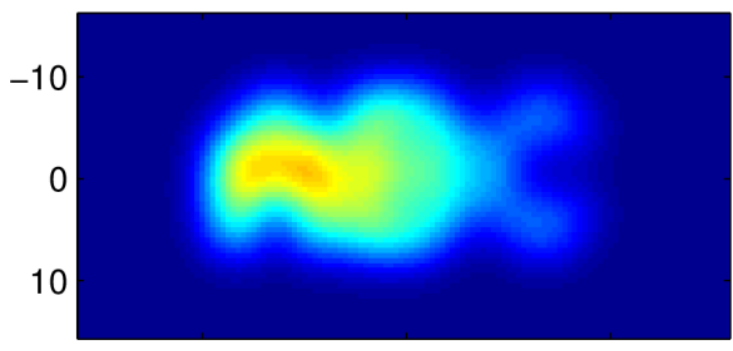

$-20$
20

d) Time $300 \mathrm{~ms}$

Fig. 4. Results from simulation of three arbitrary defects with shapes like the letters $A, B$ and $C$ and depths $0.5,1.0$ and $1.5 \mathrm{~mm}$. The scale on the $x$ and $y$-axis is in $\mathrm{mm}$.

One of the main advantages with this simplified analytical model is that the computational time is low even for complex defects such as those in figure 4. To calculate the response from these defects, with a resolution of $128 \times 128$ pixels at 20 different times, took less than $0.2 \mathrm{~s}$ on a normal laptop.

\section{Conclusion}

A model has been developed that can be used for modelling arbitrarily shaped defects in homogenous material. Since it is an analytical solution it can be computed relatively fast for any defect shape and for any point in time, without a loss of accuracy. A comparison was made to real data which showed a good agreement even though the model is for a simplified geometry. To demonstrate the possibilities of the model, the response from three defects with complex shapes was presented at different times from the initial heat pulse. Since this is a simplified model it has limitations but, as can be seen, it shows good correlation to experimental data. Some of the limitations are that it is limited to a simplified geometry of the test piece and that the initial heating needs to be even and a short pulse. The modelling of a defect as a reflector of heat works well for volumetric defects but for thin defects, such as a delamination, this might be inadequate.

\section{REFERENCES}

[1] Avdelidis N.P., Hawtin B.C., Almond D.P., "Transient thermography in the assessment of defects of aircraft composites", NDT\&E International, vol. 36, pp. 433-439, 2003.

[2] Hung Y.Y., Chen Y.S., Ng S.P., Liu L., Huang Y.H., Luk B.L., Ip R.W.L., Wu C.M.L., Chung P.S., "Review and comparison of shearography and active thermography for nondestructive evaluation". Materials Science and Engineering, vol. 64(5-6), pp. 73-112, 2009.

[3] Maldague X., Ziadi A, Klein M. "Double pulse infrared thermography". NDT\&E International vol. 37(7), pp. 559564, 2004.

[4] Reigert G., Pfleiderer K., Gerhard H., Solodov I., Busse G., "Modern Methods of NDT for Inspection of Aerospace Structures", 9th European Conference on Non Destructive Testing, ECNDT, Berlin, Germany, 2006.

[5] Maldague, X.P.V., "Theory and Practice of Infrared Technology for Nondestructive Testing". John Wiley \& sons, inc., 2001.

[6] Liu, H.-n., et al., "Detection of Defects in Thermal Barrier Coatings by Thermography Analyses". Materials Transactions, vol. 44(9), pp. 1845-1850, 2003.

[7] Omar, M.A. and Y. Zhou, "A quantitative revew of three flash thermography processing routines". Infrared Physics \& Technology, vol. 51, pp. 300-306, 2008

[8] Almond, D.P., Pickering, S.G., "An analytical study of the pulsed thermography defect detection limit" Journal of applied physics, vol. 111, pp. 093510, 2012.

[9] Almond, D.P., Lau, S.K., "Edge effects and a method of defect sizing for transient thermography," Applied Physics Letters, vol. 62, pp. 3369-3371, 1993. 